\title{
Restoration of missing data in old archives based on genetic algorithm
}

\begin{abstract}
Video restoration has been an interesting area of research for many years and still with the advent of new technologies makes it an important subject to be discussed. Blotches are common defects in old archives. They refer to a small area with an approximately uniform gray level that occurs randomly in each frame. After applying most algorithms to detect the position of blotches and also scratch which is another type of defect in the old media, in each frame of video, it is essential to correct them, in other words, we should fill the missing data with reasonable values. In this paper, we consider this task similar to an optimization problem and apply Genetic Algorithm (GA) to each frame. The current frame scans row by row and is considered as the corrupted slice of each row which is found; then, we apply the GA to fill the missing data on that special portion and the process is continued to cover the image completely. The proposed algorithm is able to remove blotches and scratches with different sizes and directions and shapes. The information of previous or next frames is not needed in this implementation. The experimental results show the restored images have good quality.
\end{abstract}

Keyword: Blotches; Scratches; Genetic algorithm; Optimization problem 